\title{
Viral cancer therapies: are they ready for combination with other immunotherapies?
}
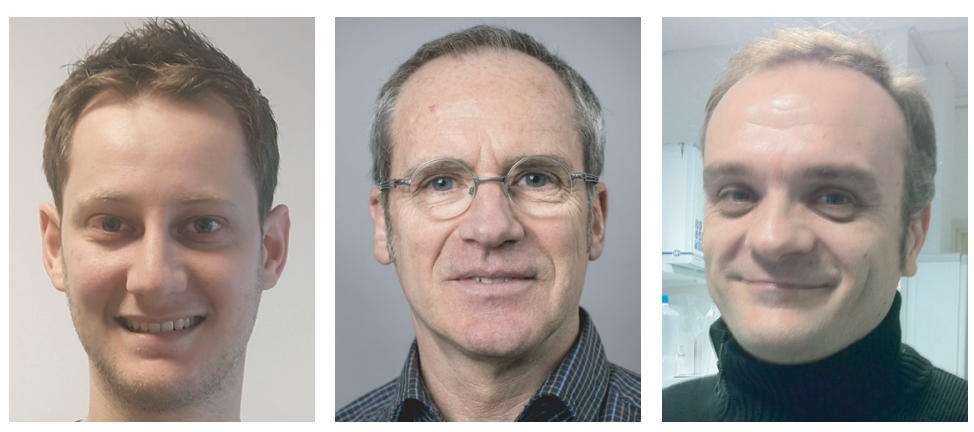

Nicolas Boisgerault*, ${ }^{*}$, Marc Grégoire' \& Jean-François Fonteneau'

First draft submitted: 21 March 2017; Accepted for publication: 24 April 2017; Published online: 4 August 2017

In the field of immunotherapy, oncolytic viruses (OVs) hold a special place at the crossroad of various antitumor mechanisms. Due to their ability to specifically target and kill cancer cells without replicating in the healthy ones, they have been extensively studied over the last two decades for their direct cytotoxic properties; tens of RNA or DNA viruses exhibiting natural or engineered oncolytic properties have been demonstrated to exert antitumor effects, in vitro, in vivo and in patients, against a wide variety of human malignancies, including aggressive cancers such as multiple myeloma, metastatic melanoma or hepatocellular carcinoma [1]. While focusing on induction of cancer cell death and tumor regressions related to viral replication, the field as a whole sometimes overlooked the role of the immunotherapeutic effects in the extremely promising results obtained with OVs at the bench or in patients. When considering data that have been accumulating over the years on OVs, results related to the basic immunological mechanisms at play are fragmented, and comprehensive understanding of how exactly the immune system participates to the therapeutic activity of these agents in patients is rather incomplete.

Nevertheless, in the recent years, the immunotherapeutic potential of OVs has been better taken into account and several ongoing clinical trials should soon provide us with critical data to improve our understanding of OVs antitumor properties. OVs appear as powerful tools when one wants to wreak havoc in immunoregulatory tumor environments in which conventional cancer therapies fail to counterbalance the action of the immunosuppressive cells responsible, in part, for tumor resistance against both treatments and the antitumor immune response. By disrupting the complex tumor organization, OVs are also anticipated to improve the efficacy of

\section{KEYWORDS}

- cancer virotherapy • immunotherapy - oncolytic virus

“Oncolytic viruses appear as powerful tools when one wants to wreak havoc in immunoregulatory tumor environments in which conventional cancer therapies fail to counterbalance the action of the immunosuppressive cells responsible, in part, for tumor resistance against both treatments and the antitumor immune response."
Future $\%$ 
other immunotherapies that would benefit from a more favorable environment to exert their own antitumor activities. As such, it is expected that $\mathrm{OV}$ replication and killing within the tumor bed would help attracting immune cells that could then be fully activated by using therapies such as pembrolizumab, nivolumab (antiPD1) or ipilimumab (anti-CTLA-4) checkpoint inhibitors (CPIs) [2], or other types of cancer immunotherapies.

When considering the first ever OV authorized for cancer treatment in the USA and in Europe, the herpesvirus Talimogene laherparepvec ( $\mathrm{T}-\mathrm{Vec}$ or Imlygic ${ }^{\circledR}$ [Amgen, CA, USA]), it is thought that its encouraging antitumor properties are to a large part related to its ability to activate the immune system in addition to direct antitumor effects [3], as suggested in particular by observations of regressing tumor lesions located at a distance from the virus injection sites. In this matter, previous results indicated the involvement of tumor-specific CD8 T cells in these effects, associated with an overall decrease of the role of different types of immunosuppressive cells such as regulatory CD4 T cells, suppressor CD8 $\mathrm{T}$ cells or myeloid-derived suppressor cells [4]. However, questions remain regarding the role of the granulocyte macrophage-colony-stimulating factor, for which T-Vec is recombinant, in the actual recruitment of human dendritic cells (DCs) at the tumor site in patients. Still, based on the positive results from the Phase III clinical trial in patients with unresectable, metastatic malignant melanoma that led to regulatory approval, T-vec has now entered clinical trials in which it is used in combination with commonly studied CPIs. The results of a recent Phase Ib trial, which will have to be confirmed in Phases II and III, already suggest an efficient combination of T-Vec and ipilimumab [5]. Ongoing clinical trials combining T-vec with nivolumab (NCT02978625) and pembrolizumab (NCT02263508, NCT02626000, NCT02965716 and NCT03069378) should also clarify the relevance of this type of combinations. Of note, whereas combination of different types of CPIs leads to noticeable toxicity, it is expected a combination of CPIs with OVs to be less toxic.

Most - if not all - OVs share immunotherapeutic properties to (re)activate antitumor immune responses [6,7]. Again, the specific immune mechanisms at play will have to be formally demonstrated but different publications report data shedding light on how OVs take part at different stages of the immune response, from induction of immunogenic cancer cell death characterized by the release of tumor-associated antigens, viral and cellular danger signals - to the priming of specific CD8 $\mathrm{T}$ cells through cross-presentation of tumor-associated antigens by antigen-presenting cells. For instance, functional immunology experiments demonstrated how oncolytic measles virus is able to cross-prime specific CD8 T-cell responses through activation of both myeloid and plasmacytoid DCs after infecting tumor cells [8]. Reovirus, through inflammatory cell killing, efficiently activates the adaptive antitumor immune response [9]. Adenoviruses have been engineered in numerous ways to take advantage of their potential immunotherapeutic capacities in different tumor models [10]. The vaccinia virus JX-594 (Pexastimogene devacirepvec), also recombinant for granulocyte macrophage-colony-stimulating factor and currently tested in a Phase III trial in patients with hepatocellular carcinoma (NCT02562755), also apparently depends on immune-mediated mechanisms for therapeutic efficacy [11].

The extreme variety of OVs and other immunotherapeutic approaches make the next few years exciting if one considers the number of combinations that could be tested to treat aggressive cancers still resisting our efforts. By acting on numerous immunological pathways that encompass both the innate and adaptive immune responses, viruses can be the key to unleash the full potential of not only immunotherapies but also other anticancer therapies for which the immune system could be of any help. As described above, OVs are expected to attract and activate different types of antigen-presenting cells (myeloid and plasmacytoid DCs, M1 macrophages, etc.) directly or through phagocytosis of OV-infected tumor cells, to limit the activity of immunoregulatory cell types (myeloid-derived suppressor cells, M2 macrophages, regulatory $\mathrm{T}$ cells, etc.) by changing the tumor microenvironment and even act on the effector phase of the immune response by activating directly cytotoxic $\mathrm{T}$ cells through different mechanisms. By concentrating our efforts on understanding exactly how OVs interact, directly or indirectly, with immune cells and drive their activation, one can envision improvements in their design and clinical use that will ultimately benefit the patients. These efforts will 
also be critical for optimal combination with other types of cancer therapies, in particular immunotherapies. By neglecting these scientific aspects, the field is at risk of missing the opportunity to revolutionize cancer treatment with to the point, rationale-based therapies. With great power comes great responsibility and to fully exploit the outstanding potential of OVs requires increased efforts at the bench to elucidate the basic immune mechanisms involved.

To date, fragmented pieces of data indicate that OVs would be relevant partners for other immunotherapies. Several clinical trials already started to study such combinatory approaches and to determine how their distinct mechanisms of action complement each other. Critical results are expected as soon as this year and may decide the future of OVs as significant players for cancer therapy. OVs are much more complex than any other type of cancer treatments and may require a longer time to set up the optimal protocols for their use in the clinic. Formal demonstration of the immunotherapeutic mechanisms at play may be the key to guarantee OVs a bright future.

\section{Financial \& competing interests disclosure}

This work was funded by grants from 'La Fondation pour la Recherche Médicale (FRM - Equipe Labellisée),' 'La Ligue Contre le Cancer' and 'L'Agence Nationale de la Recherche (ANR-16-CE18-0016).' M Grégoire and J-F Fonteneau own a patent on oncolytic measles virus. $M$ Grégoire has shares in a startup company dedicated to oncolytic viruses. The authors have no other relevant affiliations or financial involvement with any organization or entity with a financial interest in or financial conflict with the subject matter or materials discussed in the manuscript apart from those disclosed.

No writing assistance was utilized in the production of this manuscript.

\author{
“To fully exploit the \\ outstanding potential of \\ OVs requires increased \\ efforts at the bench to \\ elucidate the basic \\ immune mechanisms \\ involved."
}

\section{References}

1 Keller BA, Bell JC. Oncolytic virusesimmunotherapeutics on the rise. J. Mol. Med. (Berl.) 94(9), 979-991 (2016).

2 Rajani KR, Vile RG. Harnessing the power of onco-immunotherapy with checkpoint inhibitors. Viruses 7(11), 5889-5901 (2015).

3 Kohlhapp FJ, Kaufman HL. Molecular pathways: mechanism of action for talimogene laherparepvec, a new oncolytic virus immunotherapy. Clin. Cancer. Res. 22(5), 1048-1054 (2016).

4 Kaufman HL, Kim DW, Deraffele G, Mitcham J, Coffin RS, Kim-Schulze S. Local and distant immunity induced by intralesional vaccination with an oncolytic herpes virus encoding GM-CSF in patients with stage IIIc and IV melanoma. Ann. Surg. Oncol. 17(3), 718-730 (2010).

5 Puzanov I, Milhem MM, Minor D et al. Talimogene laherparepvec in combination with ipilimumab in previously untreated, unresectable stage IIIB-IV melanoma. J. Clin. Oncol. 34(22), 2619-2626 (2016).

6 Kaufman HL, Kohlhapp FJ, Zloza A. Oncolytic viruses: a new class of immunotherapy drugs. Nat. Rev. Drug Discov. 14(9), 642-662 (2015).

7 Zamarin D, Pesonen S. Replicationcompetent viruses as cancer immunotherapeutics: emerging clinical data. Hum. Gene Ther. 26(8), 538-549 (2015).

8 induce tumor antigen cross-presentation by human plasmacytoid dendritic cells. Clin. Cancer Res. 19(5), 1147-1158 (2013).

9 Prestwich RJ, Errington F, Ilett EJ et al. Tumor infection by oncolytic reovirus primes adaptive antitumor immunity. Clin. Cancer Res. 14(22), 7358-7366 (2008).

10 Uusi-Kerttula H, Hulin-Curtis S, Davies J, Parker AL. Oncolytic adenovirus: strategies and insights for vector design and immunooncolytic applications. Viruses 7(11), 6009-6042 (2015).

11 Breitbach CJ, Bell JC, Hwang TH, Kirn DH, Burke J. The emerging therapeutic potential of the oncolytic immunotherapeutic Pexa-Vec (JX-594). Oncolytic Virother. 4, 25-31 (2015). 